Archives de sciences sociales des religions

147 | juillet-septembre 2009

Traduire l'intraduisible

\title{
Les traductions de la Bible et l'évolution du malgache contemporain
}

Noël J. Gueunier

\section{(2) OpenEdition}

1 Journals

Édition électronique

URL : http://journals.openedition.org/assr/21385

DOI : $10.4000 /$ assr.21385

ISSN : $1777-5825$

Éditeur

Éditions de l'EHESS

Édition imprimée

Date de publication : 1 octobre 2009

Pagination : 81-103

ISBN : 978-2-7132-2217-7

ISSN : 0335-5985

\section{Référence électronique}

Noël J. Gueunier, «Les traductions de la Bible et l'évolution du malgache contemporain », Archives de sciences sociales des religions [En ligne], 147 | juillet-septembre 2009, mis en ligne le 04 décembre 2013, consulté le 19 avril 2019. URL : http://journals.openedition.org/assr/21385; DOI : 10.4000/ assr.21385 


\section{Noël J. Gueunier}

\section{Les traductions de la Bible et l'évolution du malgache contemporain}

La traduction de la Bible a exercé une influence profonde sur la constitution et la fixation de la langue malgache moderne. L'histoire culturelle du malgache est ainsi plus comparable, toutes proportions gardées, à celle de l'allemand et de l'anglais - et aussi de plusieurs autres langues de «terres de missions »-qu'à celle du français, et cela bien que le pays ait été une possession française au cours des soixante-cinq années de l'ère coloniale (1895-1960).

C'est que le processus de scripturalisation est intervenu à Madagascar avant la colonisation, dans un moment missionnaire qui a duré plus longtemps (18181895) que toute la période coloniale. Quand s'implante la domination coloniale, le trait central d'une nouvelle situation linguistique est déjà fixé : présence d'une langue écrite dotée d'outils, grammaires, dictionnaires, manuels, qui ont permis sa standardisation, et d'un corps de référence, la traduction de la Bible, achevée en 1835. L'administration coloniale a ensuite placé au-dessus de cet édifice, en position dominante, le français, langue de la colonisation et de l'administration, mais elle n'a pas pu, et ne l'a même pas sérieusement cherché, revenir sur cette situation acquise dans la société indigène.

Il est donc utile de résumer ici l'histoire des traductions et révisions de la Bible en malgache (Clark, 1887 ; Dahl, 1966 ; Munthe, 1969 ; Raison-Jourde, 1991 ; Huygues-Belrose, 2001).

\section{Le rôle des premiers traducteurs et des lettrés malgaches}

Les premiers missionnaires de la London Missionary Society (LMS) arrivent à Madagascar en 1818, et sont actifs à Tananarive dès le début des années 1820. La traduction et l'impression de la Bible en malgache sont l'un des points essentiels de leur programme. Leur première décision est le choix de l'alphabet latin pour transcrire le malgache. L'alternative - sérieusement envisagée, mais rapidement écartée - était une graphie en caractères arabes, en usage sur la côte est de Madagascar depuis des siècles, mais d'un emploi traditionnellement limité à la transcription de formulaires divinatoires et médico-magiques, et à la notation 
de traditions généalogiques et historiques. Pendant quelques années, au début du XIX siècle, le choix a été ouvert entre les deux graphies : le roi, Radama I ${ }^{\mathrm{er}}$, avait à sa cour des spécialistes des deux écritures, il avait lui-même commencé à apprendre les deux, et il avait fait l'essai de leur emploi dans les débuts d'une correspondance diplomatique. Mais, des deux groupes de conseillers religieux, devins astrologues malgaches porteurs de la graphie arabico-malgache, et missionnaires protestants porteurs de la graphie latine, c'est le second qui l'a emporté.

La manière dont le nouvel alphabet a été institué mérite qu'on s'y arrête. Les historiens ont généralement retenu l'idée que l'alphabet a été une sorte de compromis diplomatique : les consonnes de l'anglais et les voyelles du français ! Cette formule a dû être réellement prononcée dans les discussions entre le roi et les missionnaires - elle se trouve de fait dans les témoignages de l'époque. Pourtant, le principe mis en œuvre a été tout autre. Les créateurs de l'alphabet étaient conscients du fait que ni la graphie du français, ni celle de l'anglais, avec souvent pour chaque lettre plusieurs prononciations possibles, et pour chaque articulation plusieurs graphies possibles, ne pouvaient servir de modèle.

La démarche était phonologique (avant la lettre), comme le démontrent les décisions prises : on ne devait utiliser qu'autant de lettres qu'il en fallait pour en attribuer une et une seule à chaque articulation du malgache. Les lettresconsonnes $c, q, w$ et $x$ ont été abandonnées, comme faisant double emploi, et l'inventaire réduit des voyelles de la langue malgache, avec seulement quatre timbres [a], [e], [i] et [u], permettait d'abandonner aussi l'une des cinq lettresvoyelles de l'alphabet latin. Le choix du signe pour noter [u] a donné lieu à une discussion serrée, où ont été examinées successivement les possibilités de ou comme en français, de oo ou $u$ comme en anglais, et de $w$ comme en gallois, pour adopter finalement $o$, ce qui permettait de faire encore l'économie de la lettre $u$. Les seules irrégularités que la nouvelle écriture tolérait étaient le $y$ en fin de mot au lieu du $i$ simple, et le choix de la lettre $j$ pour noter [dz], seule exception à la notation par des digraphes de la série des consonnes affriquées. La rigueur phonologique du système se manifeste peut-être plus clairement encore dans la manière dont les défauts des premiers essais ont été discutés et corrigés. Ainsi, par exemple, on avait, à l'origine, noté par $k i$ un $k$ mouillé qui ne se produit qu'après la voyelle $i$. Cette graphie a été réformée en application d'un principe phonologique rigoureux : puisque la réalisation mouillée est conditionnée par la présence du $i$ avant le $k$, la consonne seule suffisait - et c'est resté la règle jusqu'à présent.

Les missionnaires sont ensuite passés à l'entreprise de traduction de la Bible - avant même d'avoir terminé l'établissement de la grammaire et du dictionnaire, qui d'après les plans initiaux auraient dû constituer l'étape suivante. C'est qu'ils étaient poussés par un sentiment d'urgence, à la fois théologique (leur mission était de mettre le message du salut entre les mains des Malgaches le plus rapidement possible) et tactique : en effet, la confiance de Radama, qui seule rendait possible leur séjour, avait fait place rapidement à la méfiance. Après la mort de 
Radama (1828), la nouvelle reine Ranavalona I ${ }^{\text {re }}$ avait d'abord paru assez favorable, mais, devenue hostile, elle s'est lancée dans une politique de persécution de la nouvelle foi. Les missionnaires se sont donc livrés à une véritable course pour arriver à terminer l'impression de la Bible avant d'être expulsés.

Ils ont en fait tout mené de front : leurs études linguistiques, les premières écoles et la traduction de la Bible. Le roi considérait les missionnaires comme une sorte d'assistants techniques chargés d'apprendre à ses sujets à lire et à écrire. Pour lui, l'école était d'intérêt immédiat : il avait besoin pour son armée et son administration d'un personnel de secrétaires, de gouverneurs et d'officiers lettrés. En fait, les missionnaires ont réussi à marier efficacement le programme de scolarisation et l'entreprise de traduction. Les exercices scolaires de traduction (de l'anglais au malgache) comprenaient des passages de la Bible, et c'est parmi les premiers élèves qu'ont été recrutés les jeunes gens - restés dans la tradition comme les «Douze»- qui aidaient au travail. Le résultat a été obtenu avec une rapidité étonnante : alors que les missionnaires commençaient à peine à se familiariser avec la langue vers 1820, le Nouveau Testament est imprimé en 1830 , et, si la Bible complète ne sort qu'en 1835, c'est en raison de la longueur des opérations de révision et d'impression, puisque le premier jet de traduction était terminé dès 1826. Cependant, l'œuvre prête à une double controverse - qui a sa source dans les ambiguités de l'attitude des missionnaires eux-mêmes : qui étaient les véritables traducteurs, les missionnaires britanniques, ou bien leurs élèves malgaches ? Et sur quel texte la traduction a-t-elle vraiment été établie : sur les textes originaux, ou sur une traduction en anglais?

La position officielle a toujours été claire : les traducteurs étaient les missionnaires eux-mêmes, et les "Douze » sont régulièrement désignés dans leur correspondance comme de simples transcribers, des secrétaires employés à mettre au propre les traductions avant l'impression. En réalité, la situation n'a pas pu être si simple, et des sons de cloche discordants se font parfois entendre dans les correspondances qui ont été conservées. Le rôle des "Douze » a certainement été bien plus important que celui de simples scribes. S'ils n'ont pas été responsables de la traduction des textes au sens propre, ils ont dû beaucoup contribuer à leur rédaction. On s'expliquerait difficilement sans cela les références, précises et nombreuses, à des réalités et à des institutions malgaches, sans parler des trouvailles idiomatiques qui parsèment le texte. On ne peut s'étendre ici sur cette discussion, mais quelques exemples donneront une idée de la richesse linguistique et culturelle de la traduction.

En Gn 38,8, il est question de Tamar, qui a d'abord été l'épouse d'Er, premier fils de Juda. Ce premier mari étant mort, Juda ordonne à son second fils Onan de prendre en mariage la fille de son frère aîné, selon la loi du lévirat. La traduction malgache donne à cet endroit : "Va chez la femme de ton frère, porte-la en "charge-sur-la-tête" (ento loloha izy), et engendre un enfant pour ton frère. " (cf. King James "Go in unto thy brother's wife, and marry her, and raise up seed to thy brother ") La notion de loloha "charge qu'on porte sur la tête ", 
désigne en effet, dans la coutume malgache, un mariage léviratique, très comparable à celui qu'institue la loi mosaïque (Dt 25,5-10). On devine ici comment l'enquête lexicale a permis à la fois de nourrir le dictionnaire d'une expression typique, et de trouver pour le passage en question la traduction techniquement la plus précise. Certaines locutions sont de simples métaphores lexicalisées dont la découverte demandait pourtant une singulière compétence, comme quand on lit, en $2 \mathrm{~S} 13,25$ : "que nous n'allions pas tous ensemble, de peur que nous ne te "brisions-la-poitrine" (mamaky tratra)", traduction qui correspond à «let us not all now go, lest we be chargeable unto thee » dans King James. La figure de la «poitrine brisée » signifie que l'on est surchargé, accablé de peine. L'emploi de certaines expressions révèle un véritable travail d'analyse. Ainsi, en Gn 15,15, passage dans lequel le Seigneur promet à Abram une digne sépulture, la version malgache de 1835 donne : "tu iras vers la paix "chez tes ancêtres" (amy ny raza'nao). " Pourtant le texte comportait une expression qui se traduirait mot à mot par "chez tes pères" (cf. King James "thou shalt go to thy fathers in peace»). Mais une traduction littérale en malgache aurait donné un résultat difficile à accepter, pour des raisons à la fois sociales et linguistiques. D'une part, la sépulture malgache n'est pas comprise comme celle des seuls "pères ", mais comme celle d'un collectif ancestral qui couvre toute l'étendue de la parenté, si bien que c'est effectivement le terme raza'nao " tes ancêtres » qui convenait ici. D'autre part, du fait de l'absence de marque du nombre en malgache une transposition terme à terme de " chez tes pères " aurait sans doute été comprise " chez ton père ", ce qui prêterait à contresens. Il est plus que probable que les missionnaires n'ont pu faire seuls de telles trouvailles.

L'attitude des missionnaires vis-à-vis de leurs collaborateurs malgaches dans les années 1820-1830 était sans doute proche de celle que montre, bien plus tard, leur collègue William Cousins dans ses souvenirs sur la "grande révision » de 1874-1887. Il écrit en effet :

«Une grande considération est due à notre petite compagnie d'assistants indigènes (our little band of native helpers); souvent quand je lis maintenant la Bible, je rencontre des tournures que nous leurs devons. Parmi ces aides (these helpers), aucun ne mérite plus d'éloges que notre ami Andrianaivoravèlona, et bien qu'il ne soit plus parmi nous physiquement, chaque fois qu'on lit la Bible dans nos temples, il nous parle encore par plus d'une expression idiomatique qui y a été introduite sur sa suggestion. » (Cousins 1897 : 118).

On note le ton protecteur : les indigènes - dont on reconnaît certes le mérite peuvent être nos " amis ", ils n'en restent pas moins des assistants, des auxiliaires. Cette attitude n'était certainement pas à cette époque une particularité des missions malgaches. Le missionnaire anglais Carey, qui avait publié au début du $\mathrm{XX}^{\mathrm{e}}$ siècle les premières traductions de la Bible en bengali et en plusieurs autres langues de l'Inde, était pour ceux de Tananarive un modèle admiré. Nous le savons parce que Kingdon, l'imprimeur de la mission quaker, avait fait à Tananarive devant l'Association des jeunes gens chrétiens une série de conférences sur la vie 
et les vertus héroïques de ce grand devancier. Or, il faisait dire au missionnaire du Bengale :

«Tout mon salaire je le dépense pour salarier une personne pour m'aider (akaramako olona hanampy aby) à traduire l'Écriture Sainte, et à en faire la correction; et je le dépense aussi à payer un professeur qui m'aide» (Kingdon, ca. 1878:15).

Nul doute que le type de relations ainsi décrit entre le traducteur missionnaire européen et son « aide » ou son "professeur » indigènes ne devait résonner d'une manière familière aux oreilles des auditeurs malgaches. Les missionnaires ne doutaient pas que c'était à eux que revenait l'œuvre originale de la traduction : les locuteurs natifs étaient des aides, certainement pas les auteurs intellectuels du travail. Nous sommes loin de l'attitude d'un Maurice Leenhardt cherchant pour la traduction du Nouveau Testament en houailou non pas une aide du catéchiste locuteur natif, mais une formulation nouvelle, en quelque sorte jaillissant des mots de la langue; mais il est vrai que nous sommes alors au $\mathrm{XX}^{\mathrm{e}}$ siècle, et que d'ailleurs les intuitions de Leenhardt sont loin d'avoir été acceptées de tous (sur la comparaison des attitudes de Carey et de Leenhardt, voir Smalley, 1991 : 50-58).

La question du texte de base sur lequel la traduction a été établie prête également à controverse. Les missionnaires ont insisté sur le fait que la traduction devait partir des textes originaux, hébreux et grecs, et non d'une traduction. Ils en avaient, en principe, la compétence. En effet, l'enseignement du séminaire de Gosport où ils avaient étudié, non seulement donnait une part importante aux études linguistiques, mais Munthe (1969) a montré qu'il était aussi caractérisé par la liberté intellectuelle des milieux protestants non conformistes vis-à-vis des normes plus conservatrices de l'Église d'Angleterre. Partant pour Madagascar, les missionnaires de la LMS étaient au courant des acquis les plus récents de la recherche exégétique. Ils avaient avec eux en particulier le Nouveau Testament grec de Griesbach, qui représentait un grand progrès dans l'établissement du texte par rapport au Textus receptus, héritier des recherches humanistes de la Renaissance, auquel se tenait encore l'Église anglicane. Et ils ont effectivement travaillé sur ce texte grec: Munthe a démontré, en suivant verset par verset les omissions et les additions de l'édition Griesbach, que la traduction malgache n'avait pas suivi le texte anglais disponible à l'époque (établi sur le Textus receptus), mais bien le texte de Griesbach. Cela signifie-t-il que la traduction a réellement été faite à partir du grec, ou bien plutôt qu'une traduction établie d'abord sur l'anglais a été ensuite soigneusement collationnée pour la mettre en accord avec le grec ? Les déclarations des missionnaires eux-mêmes laissent planer le doute. Les pages de titre du Nouveau Testament (1830) et de la Bible complète (1835) donnent des indications qui ne concordent pas. Sur la première on lit : no dikai'ny ny Misionary tamy ny teny Girika « traduit par les missionnaires de la langue grecque ", alors que sur la seconde figurent les mots : voadikia tamy ny teny Hebirio sy Girika « traduite des langues hébraïque et grecque », mais suivis de l'explication : ka nafindra ny ny Misionary, avy amy ny "London Missionary 
Society," ho teny Malagasy «et transposée [ou : "transportée"] par les Missionnaires de la LMS en langue malgache ». Cette formulation ${ }^{1}$ ne signifie-t-elle pas que la traduction proprement dite était en une langue intermédiaire (l'anglais) avant la «transposition » vers la langue malgache ? Et en quoi consistait exactement cette dernière opération ? La discordance s'explique-t-elle par des procédures différentes pour le Nouveau et pour l'Ancien Testament, dues à ce que les compétences pour l'hébreu étaient certainement plus rares que pour le grec ? Ou bien est-ce une fois de plus une trace des désaccords théoriques entre les membres de l'équipe de traduction?

La discussion n'est donc pas close, et on voit réapparaître la controverse, par exemple chez l'historien Pier Larson (travail en cours), qui insiste pour décrire le processus de traduction comme une «translation of the Bible from the English King James Version" (nous avons vu qu'en toute rigueur textuelle cette description est inexacte), et pour attribuer le titre de premiers traducteurs aux "Douze ", décrits comme "teenage Malagasy boys who had been taught English ». La controverse a un arrière-plan idéologique : insister sur le caractère original de la traduction, et sur le recours aux textes grecs et hébreux, c'est donner la place prédominante dans le travail aux missionnaires européens, les seuls dans la Tananarive des années 1820 à pouvoir lire les langues anciennes. À l'inverse, reconnaître un rôle prépondérant aux " Douze ", c'est maximiser la contribution des Malgaches - mais alors il faudrait que la traduction ait été faite sur l'anglais... La position de Larson est cohérente avec son point de vue général sur l'histoire des débuts du XIX siècle malgache : dans un de ses travaux remarqués, il proposait déjà de revoir l'interprétation généralement présentée des relations entre Radama ${ }^{\text {er }}$ et le gouverneur Maurice Farquhar. Alors que les historiens malgachisants placent l'initiative exclusivement du côté des Britanniques, Larson (2000:224-225) réévalue l'autonomie stratégique du roi malgache qui a recherché activement l'alliance étrangère et su en tirer le meilleur profit.

\section{Un moment fondateur pour la langue malgache moderne}

Un point très important pour l'avenir est la décision prise, au moment de l'impression de la Bible, sur la nature et sur le nom qui sera donné à la variété de langue choisie pour la traduction. Les pages de titre de 1830 (Nouveau Testament) et 1835 (Bible complète) portent la même indication : le Livre a été traduit ho teny Malagasy "en langue malgache ". Sur ce point, comme sur beaucoup d'autres, il y avait désaccord entre les missionnaires. Munthe (1969: 97) rapporte qu'en 1834, au moment de l'impression du livre d'Ésaïe, le titre avait d'abord été rédigé par l'un des missionnaires, Griffiths, avec l'expression teny

1. Reprise en anglais sur une page de faux-titre : "translated from the Hebrew and Greek Languages: and retranslated into the Madagascar language by the Missionaries of the "London Missionary Society.” " 
nkova "en langue hova ", et que c'est son collègue Freeman qui avait corrigé sur les épreuves teny Malagasy. Griffiths avait protesté, expliquant que tout ce qui avait été imprimé l'avait toujours été en hova. En 1834, la querelle pouvait paraître byzantine: de fait, la langue malgache que les missionnaires apprenaient, et dans laquelle ils publiaient le résultat de leurs travaux, était celle en usage à la cour de Tananarive, au cœur de cette région que les traitants européens connaissaient depuis le XVIII ${ }^{\mathrm{e}}$ siècle comme l'Ankova, le pays des Hova, et ce nom s'employait encore pour désigner les habitants de la région centrale ${ }^{2}$. Mais le choix avait une signification politique - et il s'est révélé par la suite un choix de conséquence dans le domaine sociolinguistique. La reine Ranavalona avait protesté contre l'habitude que les Européens avaient de la désigner comme "reine des Hova » (Raison-Jourde, 1991 : 33), alors qu'elle affirmait être la souveraine légitime de tout Madagascar. Malagasy était le mot choisi pour désigner les habitants de son royaume, par opposition aux étrangers du delà des mers. Présenter la Bible comme ouvrage en langue malagasy, c'était donc en faire le premier monument d'une littérature non pas locale, dialectale, mais nationale - ce qu'elle est effectivement devenue, même si en 1830-1835 le processus de standardisation de la langue, qui devait lui donner progressivement une autonomie par rapport au parler oral de l'Imerina, n'en était qu'à ses premiers pas. La question de la nature de la langue écrite : simple extension du dialecte de la région de la capitale, ou langue standardisée capable de servir de koinè à l'ensemble du pays, est devenue un serpent de mer qui réapparaît périodiquement dans la vie politique malgache, en particulier chaque fois que les autorités déplacent les limites entre l'usage du malgache et l'usage du français dans l'enseignement ${ }^{3}$. On peut donc dire que cette dispute, devenue depuis une constante de la vie politique malgache, trouve son fondement en ce moment-clé.

Les résultats de la première période missionnaire protestante ont été impressionnants du point de vue religieux, et aussi culturel. La persécution, loin de faire disparaître le tout petit noyau des croyants, l'a au contraire radicalisé, si

2. Mais il cédait la place devant un autre mot, Merina - le nom Hova se spécialisant comme celui d'un des ordres hiérarchiques de la société, les roturiers. L'emploi de Hova comme ethnonyme a persisté longtemps dans la littérature coloniale.

3. Les aménagements contradictoires se succèdent pendant la période coloniale : arrêtés de 1916 et de 1933 privilégiant le français, de 1929 et de 1951 augmentant la place du malgache (Esoavelomandroso, 1976). Après l'Indépendance, l'ampleur du mouvement de bascule augmente (en 1978, le malgache devient seule langue d'enseignement, puis en 1991 c'est le français qui est déclaré, en dépit de tout réalisme, seule langue d'enseignement). En juillet 2008, une nouvelle décision fait du malgache la langue d'enseignement dans les cinq premières années de l'enseignement primaire. À chaque étape, le même argument se retrouve dans les polémiques : donner plus de place au malgache, c'est défavoriser les « côtiers » en leur imposant une " mérinisation » linguistique. Le fait qu'entre temps la langue littéraire s'est largement différenciée du parler oral de l'Imerina, et que la vie publique, en particulier politique, se passe désormais entièrement en cette langue - y compris les discours de ceux qui dénoncent la " mérinisation »n’empêche pas la reproduction quasi rituelle des mêmes discussions. 
bien qu'après la mort de Ranavalona Ire (1861) une communauté chrétienne décidée attendait le retour des missionnaires. Parallèlement, la constitution d'une élite lettrée et d'un standard linguistique reconnu avait fourni au royaume un instrument de gouvernement : bien que l'œuvre missionnaire ait été arrêtée, cet acquis de l'écrit a été maintenu et utilisé par les scribes de l'administration royale ${ }^{4}$. Il est très remarquable que c'est aussi pendant le «Temps de l'Obscurité » (ny Tany Maizina, terme que l'historiographie ecclésiastique applique à la période des persécutions) qu'ont été écrites les premières productions littéraires malgaches. L'imprimerie étant interrompue, c'est sous forme de manuscrits qu'ont été collectés les textes qui devaient devenir ensuite, aux «Temps de la Lumière ", les classiques de la littérature malgache. Les contes, publiés en 1877 par le missionnaire luthérien Dahle (discussion récente dans Noiret, 2008), comme les traditions de l'Histoire des Rois dont les cahiers ont été copiés et publiés par le jésuite Callet à partir de 1873 (discussion dans Condominas, 1984), ne sont pas des collectes de folkoristes européens, mais, pour l'essentiel, la publication de cahiers de lettrés malgaches, élèves des missionnaires. Il en va de même de la poésie sapientielle des proverbes et des pièces de "science du verbe " hain-teny, dont certains cahiers conservés par les familles de leurs auteurs ne devaient voir le jour que plus d'un siècle après la mort de Ranavalona (DomenichiniRamiaramana, 1968, 1983).

Un langage religieux était né, à la suite de décisions qui, à l'origine, pouvaient prêter à discussion, mais qui ont aussitôt constitué un vocabulaire chrétien accepté de tous, sur lequel aucune révision ou traduction ne devait plus songer à revenir. On se limitera à un exemple. Pour traduire "Dieu ", deux termes étaient disponibles. L'un, Zanahary, semble renvoyer à la notion de "Créateur ». L'autre, Andriamanitra, est un composé dont le sens littéral est «Seigneur qui embaume, qui sent bon" (Dahl, 1992). Les deux pouvaient s'appliquer à la divinité suprême, mais aucun des deux n'était spécialement lié à l'idée de Dieu unique, et en particulier l'un et l'autre étaient employés pour invoquer les palladiums royaux de l'Imerina - ces objets sacrés que les missionnaires avaient identifiés à des « idoles ». Entre les deux noms, les missionnaires protestants ont visiblement hésité puisque, dans leurs cantiques, ils ont souvent utilisé Zanahary. Mais dans la traduction de la Bible, ils ont employé seulement Andriamanitra. Le résultat a

4. Cela justifie le terme de "malgache officiel » malagasy ôfisialy, employé aujourd'hui pour désigner la langue standard (Rajaonarimanana, 1995 : 12, 218). Si cet article, consacré aux traductions de la Bible, s'intéresse surtout à l'apport des Églises, il serait exagéré de leur attribuer tout le mérite de la construction de la langue moderne. La priorité revient même au côté officiel puisque les débuts d'une chancellerie royale datent précisément de 1816-1817, un an avant l'arrivée des missionnaires : on a conservé des lettres de ces années, envoyées par le roi Radama aux autorités de l'Île Maurice, en graphie arabe. La « langue officielle » y est encore influencée par les dialectes de l'est qui étaient ceux des scribes arabico-malgaches, influence qui devait cesser après l'adoption des caractères latins (texte et discussion dans Munthe et al. 1976). Par la suite, l'usage officiel n'a fait que se développer, du temps du Royaume, et a été maintenu par la Colonie pour l'administration et la justice indigènes. 
été qu'en malgache moderne s'est construit une opposition entre Andriamanitra, perçu aujourd'hui comme le mot " chrétien », et Zanahary qui tend à s'identifier aux conceptions tournant autour du culte des ancêtres. Les catholiques, qui dans les premiers temps avaient privilégié Zanahary, devaient rapidement "se convertir » eux aussi à Andriamanitra.

\section{Le travail des réviseurs et les nouvelles traductions}

On passera plus rapidement sur l'histoire des révisions et des traductions plus récentes ${ }^{5}$, dont l'importance linguistique et culturelle a été moindre. La première révision (1855-1865), connue sous le nom de son principal artisan, Griffiths, a la particularité d'avoir été produite en Angleterre, alors que les missionnaires, chassés par la reine, attendaient l'occasion de retourner à Madagascar. Elle n'a pas été beaucoup appréciée. On lui a reproché des erreurs grammaticales qu'expliquent sans doute les conditions de la rédaction, loin du contact avec les locuteurs. Mais elle a aussi été critiquée pour d'autres raisons, en fait pour des choix originaux qui rétrospectivement peuvent paraître intéressants. D'une part, elle poursuivait la recherche d'expressions typiquement malgaches qui avait caractérisé la rédaction de 1835. Dans un souci de cohérence, elle avait étendu l'emploi du mot razana "ancêtres " - dont nous avons parlé - à des passages, comme en 1R 19,4, où la B.1835 avait gardé une traduction littérale par ny ray «les pères ». De même, elle étend la figure de «briser la poitrine » à des contextes où l'idée d' " être à charge " avait été rendue dans la B.1835 par une expression moins idiomatique (en 2Co 11,9). Dans les cas de ce genre, elle a été, en général, suivie par les révisions ultérieures, sauf parfois par la B.2005 qui préfère souvent une expression plus prosaïque.

Mais la version de Griffiths est surtout originale en ce qu'elle avait recherché systématiquement des transpositions dans les termes de la société malgache, là où la B.1835 se croyait obligée de décrire des réalités du monde méditerranéen antique. C'est particulièrement clair dans le domaine des noms et du symbolisme des plantes. Ainsi, en Gn 30,14-16, on voit Rachel disputer à sa co-épouse Léa des mandragores, fruits des champs à l'odeur exquise réputés pour leur vertu de remède aphrodisiaque et génésique. Alors que la B.1835 avait simplement emprunté le mot anglais mandrakes - hésitant d'ailleurs sur son adaptation malgache puisqu'il était écrit tantôt mandraky tantôt mandrakisy - Griffiths transpose hardiment : il remplace par des voantango, des melons odoriférants, aptes à recouvrir un symbolisme voisin. Avec cohérence, la traduction par voantango est maintenue dans l'autre endroit où figurent les mandragores, en Ct 7,13.

5. Les références aux versions seront données par les dates d'édition : B.1835 pour la première version protestante ; B.1865 pour la révision de Griffiths ; B.1909 pour la version reçue des Églises protestantes ; B.1938 pour la traduction catholique ; B.2005 pour la traduction interconfessionnelle. 
Toujours dans le Cantique, quand le bien-aimé est comparé à un "pommier parmi les arbres de la forêt » (Ct 2,3), c'est l'anglais ( " the apple tree among the trees of the wood ", King James) qui avait inspiré le premier traducteur en lui dictant l'adaptation malgache apily. B.1865 le remplace par un citronnier (voasary), arbre élégant et de bonne odeur, cité dans des contextes comparables dans la poésie orale malgache. Le procédé n'est pas complètement systématique : Griffiths, pas plus qu'aucun des autres traducteurs, n'a proposé de remplacer l'olivier (oliva) par quelque autre oléagineux présent dans la culture locale ${ }^{6}$. Ces propositions de Griffiths, jugées sans doute trop audacieuses, seront écartées, avec beaucoup d'autres, dans les révisions suivantes qui reviennent à des emprunts à l'anglais ou au français, ou parfois à l'original hébreu, les mandragores devenant mandragora, mandragôra (B.1938, B.2005) ou dodaima (B.1909), et le pommier paoma (B.1909, B.2005) ou pôma (B.1938). (Sur ces équivalences botaniques, voir Gueunier, Solo Raharinjanahary, 2007.)

Griffiths avait mené un travail selon des principes rigoureux dans le domaine des noms propres. Pour les noms des livres bibliques, qui dans la B.1835 avaient été tout bonnement repris de l'anglais avec adaptation phonologique (Jenesisy, Ekisaodiosy, Levitikiosy), il proposait de véritables traductions par des mots signifiant "Création ", "Sortie ", "Sacrificature ", solutions qui ne seront reprises par aucune autre version. Pour les noms de lieux et de personnes, il adoptait le principe d'une adaptation rigoureuse des noms originaux grecs et hébreux, alors que B.1835 avait souvent décalqué les formes anglaises. Ainsi disparaissaient Mosesy, Jesosy, Petera..., remplacés par Mose, Iesio, Petro... Seuls quelques noms, considérés comme trop bien intégrés dans l'usage malgache pour pouvoir être changés, gardaient leur prononciation d'origine anglaise: c'était le cas notamment de "Christ ", Kraisty, qui cependant, pour se conformer à la règle du malgache excluant tout contact de consonnes, s'écrivait maintenant Kiraisity ${ }^{7}$. La plupart de ces réformes ont été abandonnées dans les versions suivantes, et nous verrons que la question de la traduction des noms propres est devenue le principal sujet de controverse dans les discussions sur les révisions ultérieures, faisant passer à l'arrière-plan des points bien plus importants.

Sur quelques points d'intérêt théologique, la traduction de Griffiths montrait également un souci de clarification, et même quelque audace. Un choix audacieux portait ainsi sur l'expression de la notion de "vierge ». Dès les premiers essais de prédication en malgache, du temps de la mission catholique de Fort-Dauphin au XVII siècle, la manière de rendre cette idée avait posé un

6. Il n'était pas non plus entièrement nouveau puisque, dès B.1835, le blé avait été remplacé par le riz (vary), et le lin par le chanvre (rongony). Ces adaptations ont été maintenues par les versions ultérieures - y compris la seconde, qui pourtant provoque aujourd'hui quelque gêne puisque, pour le lecteur contemporain, le mot rongony ne peut plus guère désigner que le hachich.

7. Il deviendra par la suite Kristy, abandonnant la diphtongue [ai] de l'anglais, mais retrouvant le contact de consonnes [st]. 
problème ${ }^{8}$. Dans la B.1835, la solution adoptée avait été un emprunt à l'anglais virjina, qui avait été employé dans Lc 1,27 «la virjina prise en mariage par un homme, du nom de Joseph », et bien sûr dans Mt 1,23 «voici : une virjina sera enceinte, et elle mettra au monde un enfant mâle ». Comme on le faisait souvent dans les traductions du temps, cette solution avait aussi été reportée sur le texte d'Ésaïe dont ce passage de l'évangile est une citation. On obtenait ainsi une concordance parfaite entre les passages de l'Ancien et du Nouveau Testament, en dépit du fait que le mot du texte hébreu d'Ésaïe ne comporte pas l'idée de virginité. La B.1865 prenait la situation au revers : il renonçait à l'emprunt, et le remplaçait par des mots existant dans le vocabulaire de base: en Lc 1,27 par zatovo-vavy mot à mot «jeune personne de sexe féminin, jeune fille» et en Mt 1,23 par le terme voisin mpitovo-vavy " célibataire de sexe féminin ». Les deux termes comportent l'idée de jeunesse et de célibat, mais pas nécessairement de virginité. Le problème de la concordance avec le texte d'Ésaie était ainsi réglé de lui-même... mais au détriment de la doctrine traditionnelle de la virginité de Marie. Là encore, la proposition n'aura pas de succès et les versions B.1909 et B.1938 reviennent à virjina, ou virjiny. Sous cette dernière forme, le mot est d'ailleurs devenu tout à fait courant dans la langue moderne.

Autant la révision de Griffiths était ambitieuse, et prenait le risque de bouleverser une tradition à peine fixée, autant les versions suivantes ont été prudentes - en fait essentiellement conservatrices. Une " petite » révision en 1871, puis une "grande ", faite par une commission qui a travaillé de 1874 à 1887, ont conduit progressivement à la version de 1908-1909, qui est devenue la Bible reçue des Églises protestantes, restée en usage jusqu'à présent. L'orthographe a été perfectionnée, mais sans que ses règles de base soient modifiées. Les dernières incertitudes morphologiques et syntaxiques ont été éliminées, donnant à la langue standard actuelle sa forme définitive. Le travail des réviseurs sur le texte a apporté de nombreuses améliorations grammaticales et stylistiques qui ont permis, de l'avis des lecteurs, de rendre les énoncés " plus malgaches ». Dans certains cas, les corrections ont permis de gommer les dernières scories dues à la connaissance imparfaite de la langue qu'avaient les missionnaires. Mais il n'est pas toujours facile de savoir dans quels cas les modifications représentaient la correction d'erreurs de langue, et dans quels cas on avait affaire plutôt à des tournures archaïques que la standardisation a fait disparaître.

Parmi les changements grammaticaux les plus notables, on note la fixation des règles d'emploi des articles ; en particulier le nom «Dieu », pour lequel il y

8. Non pas, comme on le dit parfois, que la notion n'ait pas pu s'exprimer en malgache, mais - et c'était sans doute plus grave pour les prédicateurs - parce que les expressions disponibles rendaient un sens plutôt dépréciatif : les termes retenus par le Catéchisme de Flacourt donnent le sens littéral de « réticent(e), qui n'ose pas, ne veut pas faire quelque chose » ou bien de «pas atteinte par l'homme» (Gueunier, 1996, 1997). 
avait incertitude, Andriamanitra ou ny Andriamanitra, perd définitivement son article. Le jeu des particules et conjonctions est revu méticuleusement, avec le souci de faire disparaître la répétition inlassable du ary "et ", qui marquait les textes de la première traduction. Il faut remarquer que ce "défaut " ne tenait pas toujours à un calque des textes originaux grecs et hébreux, et pas d'avantage à un calque de l'anglais de King James. C'est bien plutôt l'abandon d'un trait d'une langue encore marquée par l'oralité. Les rédacteurs sont en train d'inventer la langue malgache écrite. Nous pouvons alors relativiser l'importance des querelles dont nous avons parlé : peu importe sans doute si les principaux traducteurs étaient les Britanniques (qui avaient la direction des opérations de révision), ou les Malgaches (qui guidaient leurs efforts linguistiques) : ils ont participé ensemble à ces changements qui, à partir d'un parler oral, construisent une langue littéraire. Il est frappant de remarquer que le même type de changements s'observe entre les deux versions de cet autre classique de la langue malgache que sont les Anganon'ny Ntaolo ("Contes des Anciens »). La première édition, publiée en 1877 par L. Dahle sous le titre Specimens of Malagasy Foklore, reproduisait les textes tels qu'ils avaient été transcrits par les premiers lettrés. L'édition revue donnée en 1909 par un autre missionnaire, J. Sims, applique aux textes les mêmes principes d'une " belle langue " que, dans le même temps, les réviseurs appliquaient à la Bible.

Les catholiques avaient déjà, à la fin du XIXe siècle, des traductions partielles (les évangiles et épîtres des dimanches, puis le Nouveau Testament complet; et on avait également donné la priorité à Judith, Tobie et Maccabées, évidemment pour marquer la différence avec les protestants qui ne retiennent pas ces livres dans leur canon). Mais ils ont attendu 1938 pour donner une traduction complète de la Bible, certainement en raison de leurs réticences vis-à-vis de la lecture des Écritures par les laïcs, réticences qui sont encore explicitement mentionnées dans la préface.

\section{Des convergences interconfessionnelles à une version œcuménique}

Cependant, malgré l'atmosphère de rivalité exacerbée entre les confessions, le plus remarquable est que les catholiques ont pour l'essentiel accepté les choix faits par leurs prédécesseurs. C'est le cas d'abord des choix graphiques. Le projet de constituer ce qui aurait pu devenir une "orthographe catholique " et une " grammaire catholique » avait bien existé. L'abbé Dalmond, missionnaire aux "petites îles » (Sainte Marie et Nosy Be), points d'appui catholiques en attendant que s'ouvre la Grande Terre, a publié, dans les années 1840, vocabulaire, catéchisme, histoire sainte et cantiques, en refusant explicitement de se servir des livres des protestants. Il ne voulait pas recourir "à des ouvrages composés par des hérétiques, et écrits dans un idiome tout différent de celui des côtes ». Mais cette 
position a été vite abandonnée, et ses successeurs se sont ralliés à l'orthographe des hérétiques... revirement inévitable, puisque les règles établies à Tananarive étaient devenues celles d'une langue écrite commune (Gueunier, Rasoloniaina, 2005-2006).

Du côté du lexique, nous avons vu que les choix de base ne pouvaient plus guère être remis en question. L'idée est pourtant assez répandue dans le public malgache qu'il y a dans le texte de 1938 un "langage catholique » immédiatement reconnaissable. Elle s'appuie surtout sur la question emblématique des noms propres, où les catholiques empruntent au français ou au latin (Moizy, Piera, Joany) plutôt qu'à l'anglais (Mosesy, Petera, Jaona). Pour le reste, il y a un petit nombre d'expressions qui suffisent à jouer le rôle de marques confessionnelles. La plus connue se trouve dans la traduction de la demande " Ne nous soumets pas à la tentation » dans le Notre Père. À ces endroits (Mt 6,13 et Lc 11,4), les protestants ont ny fakam-panaby : litt. " la prise d'esprit » (le fait que l'esprit est vaincu, subjugué par le tentateur), alors que les catholiques ont ny fitaoman-dratsy : litt. "l'entraînement au mal ». En fait, il ne s'agit pas réellement d'une divergence de traduction, plutôt d'une transposition de la forme traditionnelle du Notre Père du catéchisme français - dans laquelle il s'agit comme on sait d'atténuer l'expression pour éviter aux âmes simples le scandale de penser que Dieu lui-même cherche à "induire en tentation ». En effet, dans B.1938, l'idée de «tentation » (grec peirasmon) n'est rendue par le " mot catholique " fitaoman-dratsy que dans le Notre Père. Partout ailleurs, dans les nombreux passages où il est question de la "tentation ", la traduction catholique garde le «mot protestant» fakam-panaby. Dans bien des cas, c'est plutôt par un souci d'amélioration stylistique que la traduction catholique se distingue des versions protestantes. Bien qu'en principe il s'agisse d'une traduction indépendante, et non d'une révision, le lecteur garde souvent l'impression que le traducteur avait sous les yeux la version concurrente des protestants, et qu'il a simplement cherché à faire mieux en travaillant la prosodie, pour obtenir des phrases plus coulantes, et mieux adaptées à la lecture en public.

Dès les années 1960-1970, ont vu le jour des projets de révision de la version catholique, qui n'ont conduit qu'à des traductions partielles, et un projet de traduction œecuménique, ou interconfessionnelle, associant catholiques, anglicans et protestants. Les progrès ont été très lents - le Nouveau Testament étant imprimé en 1991, et la Bible complète en 2005. L'entreprise a été laborieuse, l'obstacle le plus évident étant, comme toujours, la question des noms propres, obstacle si difficile à surmonter qu'on a dû d'abord imprimer pour un premier fascicule publié à titre d'essai, une «traduction œcuménique, version protestante ", et une " traduction œcuménique, version catholique ", qui ne différaient que par les noms propres! Ce n'est qu'ensuite que la commission a réussi à se mettre d'accord sur une solution minutieusement négociée : quelques noms ont été refondus, d'autres repris à l'une ou l'autre des anciennes versions. Mais 
l'ambition de la nouvelle version dépasse de beaucoup cet aspect de compromis interconfessionnel ${ }^{9}$. S'agissant du vocabulaire, il ne pouvait être question de bouleverser le "langage chrétien " dont on a parlé. Au contraire, l'ensemble de cet héritage a été maintenu, même là où il était discutable, comme dans le cas du terme choisi pour "prophète » mpaminany, un mot qui signifie litt. " devineur ", mais qui est lié maintenant en malgache principalement aux contextes bibliques. Il fallait cependant gommer certains mots transmis de version en version depuis la première traduction, et qui étaient devenus si archaïques qu'ils finissaient par gêner la compréhension. Ce point était assez facile. Un exemple se trouve dans le verset du Magnificat, Lc 1,48, où la "servante » se disait, dans toutes les versions, d'un mot de l'ancienne langue, euphémisme courant aux temps de l'esclavage qui appelait les serviteurs et servantes des " enfants ", ankizy (comme fait en d'autres endroits le grec pais). Le mot est aujourd'hui désuet en ce sens, et pour les nouvelles générations il fait confusion avec les véritables enfants. La nouvelle version est beaucoup plus claire en le remplaçant par un nom d'agent formé régulièrement sur le verbe "servir » (et qui est d'ailleurs dans la langue moderne le nouvel euphémisme pour dire « esclave»).

Au-delà des questions de lexique, l'équipe de traduction s'est explicitement inspirée de la théorie de l'«équivalence dynamique » prônée par E.A. Nida et ses disciples de l'Alliance Biblique Universelle. Ce principe suppose surtout qu'on renonce aux calques des structures des langues originales qui produisent dans la langue d'arrivée des énoncés inusités, ou au moins étranges. Le même verset du Magnificat en donnait un exemple dans les anciennes versions qui disaient, mot à mot : "il a regardé la bassesse de position de son enfant [c'est-à-dire de sa servante] »; recherchant une expression plus naturelle, la nouvelle version donne à peu près : "il m’a regardée, moi sa servante de rien ». L'application de ces principes devait amener les traducteurs à essayer de rendre l'expression plus coulante, plus "malgache »- ce qui était déjà, on l'a vu, le souci constant de leurs prédécesseurs. Ils ont ainsi revu l'orientation syntaxique de nombreuses phrases, pour faire prévaloir, chaque fois que c'était possible, et chaque fois que les versions précédentes ne l'avaient pas encore fait, la préférence du malgache pour l'expression à la voix passive ou à la voix circonstancielle (dans laquelle le sujet grammatical de l'énoncé est une circonstance du procès, le lieu, le temps, la manière, etc.) Mais, d'un autre côté, le souci de simplifier l'expression, et la méfiance vis-à-vis de la transposition littérale des figures "bibliques », caractéristiques des théories de Nida, ont conduit parfois à abandonner des tournures qui se trouvaient «coller» fort bien aux points de vue malgaches. Ainsi, dans

9. Les apports de cette version ne se résument pas aux quelques traits qui seront mentionnés ici. D'une manière générale, elle s'efforce de tenir compte de toutes les recherches nouvelles. En particulier pour ce qui est du tétragramme divin YHWH, elle renonce aux lectures - qui avaient été «modernes» en leur temps Jehovah (B.1835, B.1909) et Iaveh (B.1938) - pour revenir avec ny Tompo («le Seigneur») à une solution conforme à la fois à la lecture juive (Adonai) et aux traditions de traduction en grec et en latin. 
le cantique de Zacharie, Lc 1,69, les anciennes versions disaient : « il a dressé la corne du salut pour nous ». La métaphore a été gommée au profit d'une phrase plus explicite qui se traduit: "c'est un sauveur puissant qu'il a élevé pour nous »... Et pourtant l'image de la corne comme expression de la puissance est bien attestée dans la culture malgache; le roi Radama appelait son armée, qui était la force de son État : "les cornes du royaume »(Callet, 1908:1076), et l'expression parle aujourd'hui encore dans une société où les traditions pastorales ne sont pas près de s'effacer. Il n'était sans doute guère justifié de supprimer ici la figure biblique. En fait, dans plusieurs cas, la version B.2005 suit de si près les recommandations des équipes internationales d'appui à la traduction qu'elle donne l'impression de ne plus calquer le grec ou l'hébreu, mais de calquer les solutions expérimentées en anglais ou en français dans Good News for Modern Man ou Bonnes Nouvelles Aujourd'hui... Le souci d'éviter des obscurités et des confusions en recourant à ce que la théorie de Nida appelle des «périphrases légitimes » (c'est-à-dire des périphrases qui ne sont pas des commentaires mais l'explicitation $\mathrm{du}$ sens propre $\mathrm{du}$ mot) conduit parfois à des formules embrouillées ou tautologiques comme dans Rm 7,25 quand la «chair » des traductions anciennes devient "le fait que je suis un homme pécheur ", et que l'apôtre enseigne "je suis réduit à l'esclavage par le pouvoir du péché, par le fait que je suis un homme pécheur ».

On note aussi dans la nouvelle version une tendance à l'atténuation, justifiée par le souci d'éviter de fausses interprétations, mais qui risque d'affadir singulièrement le texte. C'est dans le Cantique qu'on en trouve les plus beaux exemples. Dans Ct 1,2, là où les versions B.1909 et B.1938 avaient l'équivalent du classique "qu'il me baise (hanoroka) des baisers (fanorohana) de sa bouche », B.2005 remplace par «qu'il m'embrasse (hanoroka) vraiment fort (mafy tokoa) »; on a évité, conformément à la théorie, le sémitisme typique de "l'objet interne ", mais le moins qu'on puisse dire est que l'expression n'en a pas été renforcée ${ }^{10}$. En Ct 1,5, là où la Belle disait selon B.1835 et B.1938 «Je suis noire (mainty) mais je suis belle... », et selon B.1909 "J'ai noirci [ou : je tourne au noir, mivalomainty], mais je suis belle... ", la nouvelle version lui fait dire : "Je suis de peau un peu foncée (zarazara), mais bien faite... ». Quand on sait la signification sociale que revêtent en malgache les termes désignant les couleurs de peau, on ne peut qu'être frappé par le remplacement de mainty «noir " (qui s'applique aux descendants des anciens esclaves, encore victimes dans la société actuelle de graves discriminations) par zarazara, qui signifie " assez foncé, ni vraiment blanc, ni vraiment noir ». Ici, ce sont les implications sociales du texte qui risquent de paraître atténuées par l'affaiblissement de l'expression, même s'il n'est pas sûr du tout que telle était l'intention des traducteurs.

10. Ici, l'atténuation a été continue, puisque B.1835 avait l'équivalent de "qu'il me suce (hitsentsitra) des suçons (fitsentsefana) de sa bouche ». C'était avant que la mode des baisers à l'européenne ne se diffuse à Madagascar. 
D’une manière générale, la traduction interconfessionnelle, enfin complète après plus de trente ans de travaux, n'a pas enthousiasmé les usagers. On lui reproche ses longueurs, son prosaïsme. Elle se heurte certainement à la sacralisation dans chaque Église des textes reçus. Certains ont craint que sa parution ne signifie que le nouveau texte, devenant officiel, ne vienne abolir les anciennes versions. Surtout, les progrès de l'œuménisme, très réels si on se reporte aux vifs antagonismes qui étaient de règle avant les années 1960-1970, sont arrivés depuis à une situation stationnaire : les appareils des différentes Églises collaborent régulièrement, surtout en cas de crise avec le pouvoir politique, et les fidèles se sont habitués aux célébrations interconfessionnelles, présidées par des clercs ou purement familiales, notamment pour les funérailles, qui sont toujours un grand moment de la vie sociale. Mais il n'est plus question de communion, plutôt de coexistence; quand on est ensemble, on a pris l'habitude d'alterner textes " protestants » et "catholiques », ce qui a l'avantage de ménager l'identité de chaque groupe. Il n'est donc pas étonnant que la parution de la traduction œcuménique ait coïncidé avec l'annonce que, chacun de leur côté, catholiques et protestants préparaient une révision de leur propre version. La révision catholique, limitée à des mises au point de détail, est parue en 2006.

\section{Langage biblique et vie moderne}

On a montré plus haut les adaptations remarquables qui, en conférant des sens nouveaux à des termes sortis des contextes de l'ancienne société, ont contribué à donner une coloration malgache aux textes de l'Écriture. Il faut maintenant décrire un mouvement qui en est d'une certaine manière l'inverse : une fois le corpus biblique établi et reçu, il a pu servir à son tour de matériau pour forger une terminologie moderne dans plusieurs domaines, en particulier ceux des relations sociales et politiques. Ce travail a été celui des écrivains, dont la grande période se situe au cœur de la colonisation et jusqu'aux débuts de l'indépendance $^{11}$, mais aussi - et on y retrouve souvent les mêmes noms - du journalisme, des manuels scolaires, sans oublier les rédacteurs de l'administration coloniale elle-même, qui employait la langue malgache officielle pour beaucoup de ses relations avec les "indigènes". Il en résulte cette abondance d'expressions bibliques, qui vont de la métaphore lexicalisée (le «meuble fragile », la femme, glissement sémantique à partir d'un terme qui traduisait le "vase fragile » de $1 \mathrm{P}$ 3,7 ), au proverbe ( faire retraite de Juifs ", d'après le recul des accusateurs de la femme adultère, Jn 8,9), et au slogan politique ("abattre les murailles de Jéricho ", d'après Jos 6,1-20), qui ont attiré l'attention des observateurs de la vie malgache (Spindler, 1992 ; Gueunier, 2004). Ainsi la langue moderne peut

11. Voir les meilleures données en français sur cette question dans les articles de Delval, Rajaonarimanana et Vérin dans le Dictionnaire Universel des Littératures (1994). 
paraître au moins autant un "malgache religieux» qu'un «malgache officiel » ${ }^{12}$ : c'est le langage du politique qui se met en dépendance du langage du religieux bien plus que l'inverse, d'autant que les Églises gagnent régulièrement en influence ce que perd l'État, affaibli par l'échec économique et par sa quasi mise en tutelle par les organismes financiers internationaux.

Plus significatif encore a été le remodelage du lexique lui-même. C’est de leurs usages dans les contextes bibliques que les deux termes foko et firenena, qui désignaient originellement des groupes de descendance, et relevaient donc du registre de la parenté, en sont venus à se spécialiser respectivement au sens d' " ethnie » (régionale) et de " nation » (exaltée dans son unité, s'opposant précisément aux dissensions "ethniques »). Depuis l'époque coloniale, et jusqu'aux récents mouvements populaires de la capitale, les souvenirs de la Genèse ( « Je ferai de toi une grande firenena ", Gn 12,2), de l'Exode ("fais monter cette firenena », Ex 33,12) et de l'Apocalypse ( «beaucoup de peuples, de firenena, de langues et de rois " Ap 10,11) colorent l'idée de la nation. La notion de progrès (fandrosoana), caractéristique de l'idéologie politique malgache moderne, rappelle les théories développementalistes des débuts de l'indépendance, mais surtout une idéologie missionnaire qui associait les lumières de la foi à celles de l'instruction et de la civilisation. Du même radical (roso) on tire les mots qui désignent le progrès technique, et la progression du néophyte vers le baptême. Ce même radical rend l'idée d' "édification " par exemple en 1Co 8,1, ce que le français rend ordinairement par "la connaissance enfle, mais la charité édifie » se dit en malgache depuis la B.1909 : " la connaissance fait enfler, mais l'amour fait progresser (mampandroso) ». Là où le texte biblique avait une métaphore du registre de l'architecture, oikodomein " édifier, construire la maison ", les traductions malgaches ont choisi une image du domaine du déplacement, « faire avancer ».

Parfois, le recours à la Bible comme source de néologismes est explicite. Lorsque, après la révolution étudiante de 1972, le besoin d'acclimater les notions du marxisme s'est fait sentir, un des intellectuels engagés de l'époque a confié avoir découvert dans les Psaumes le terme pour rendre l'idée de classe sociale. C'était saranga, un mot de l'ancienne langue qui rendait l'idée de " rang, dignité ", dans Ps 55,12-13, selon la B.1909 : "Ce n'est point un ennemi qui me dénigre mais c'est toi, un homme de même saranga que moi, mon propre ami et camarade ». Un fois lancé, le néologisme a connu un succès immédiat, et le sens «marxiste» est aujourd'hui son emploi principal ${ }^{13}$.

C'est ce langage commun qui explique sans doute le paradoxe de la situation culturelle actuelle : le recul, ou le refroidissement, de l'œcuménisme institutionnel se concilie assez bien dans la vie avec une association des traditions catholique

12. Selon le nom qui lui est généralement donné, $c f$. plus haut note 4 .

13. Confidences reçues par F. Raison-Jourde (communication personnelle). 
et protestante - qui s'étend d'ailleurs souvent également aux cultes ancestraux. Là où le sociologue pouvait être tenté de chercher un syncrétisme, on verra plutôt négociation entre des traditions qui restent distinctes ${ }^{14}$. Cet « œuménisme » informel, incluant un accommodement avec le rituel ancestral, est certes repoussé vigoureusement par tous ceux qui adhèrent aux mouvements plus radicaux, de tendances revivaliste ou pentecôtiste, pour qui tout compromis avec les «païens » et avec «le train de ce monde » est inconcevable. Des affrontements peuvent se produire, parfois allant jusqu'à la violence - on a vu il y a peu des incendies de lieux de cultes «païens » revendiqués par les mouvements chrétiens de Réveil, sur le modèle de la destruction des «idoles » par la Reine nouvellement convertie en 1869. Mais le langage dans lequel s'expriment ces tendances opposées est largement unifié. Une étude récente montre comment les références bibliques, surtout vétérotestamentaires, sont utilisées aussi bien dans le rituel d'un mouvement de Réveil qui a restitué au profit de sa Prophétesse, d'après les descriptions de l'Exode et du Deutéronome, les rites de l'intronisation du grand-prêtre, que dans les rituels de prières sur les hauts lieux royaux, ou dans les pratiques d'un guérisseur, qui ne tient pas comme la plupart de ses confrères sa connaissance des plantes d'une tradition familiale, ou d'un apprentissage, mais d'un système complexe de correspondances qui lui permet de remplacer par des espèces existant dans le pays les plantes mentionnées dans l'Écriture (Razafindrakoto, 2006). Ainsi les oppositions, même violentes, n'empêchent pas les uns comme les autres de puiser à la même symbolique biblique.

Noël J. GUEUNIER

Université Marc Bloch - Strasbourg noel.gueunier@wanadoo.fr

14. On trouve description et analyse de ce réveil des cultes ancestraux dans deux ouvrages récents, Rakotomalala et al., 2001, et Blanchy et al., 2006. 


\section{Bibliographie}

Blanchy Sophie, Rakotoarisoa Jean-Aimé, Beaujard Philippe, Radimilahy Chantal, (dirs.), 2006, Les dieux au service du peuple: Itinéraires religieux, médiations, syncrétisme à Madagascar, Paris, Karthala.

CAllet François, 1873-1902, Tantara ny Andriana eto Madagascar. Documents historiques d'après les manuscrits malgaches, Antananarivo, Presy Katolika, 4 vol. [Réédit. Académie Malgache 1908 ; trad. fr., Histoire des Rois, (trad. Chapus \& Ratsimba), 1953-1978.]

Clark Henry E., 1887, Tantaran'ny Fiangonana eto Madagasikara [Histoire de l'Église à Madagascar], Antananarivo, FFMA.

Condominas Georges, 1984, "Deux grands ethnologues pratiquement inconnus... ", in Rupp-Eisenreich B., (dir.), Histoires de l'anthropologie, Paris, Klincksieck, pp. 161-209.

Cousins William E., 1897, "Reminiscences of Bible Revision ", Antananarivo Annual, 21, pp. 117-119.

DAHL Otto C., 1992, «Zanahary, Andriananahary, Andriamanitra. Désignation des êtres divins en malgache ", in Condominas G., (éd.), Disciplines croisées, Paris, Éditions de l'École des Hautes Études en Sciences Sociales, pp. 99-114.

-, 1966, Les débuts de l'orthographe malgache. Oslo, Universitetsforlaget.

DAHLE Lars, 1877, Specimens of Malagasy Folklore, Antananarivo, A. Kingdon. [Révisé par J. Sims, 1908, et constamment réimprimé depuis ; trad. fr., Contes des Aïeux malgaches. Anganon'ny Ntaolo, (trad. Dorian \& Molet), Paris, Institut des Langues et Civilisations Orientales, 1992.]

Dictionnaire Universel des Littératures, 1994, (Béatrice Didier, dir.), Paris, Presses Universitaires de France, [Articles de: R. Delval sur "Théâtre malgache"p. 3797 ; N. Rajaonarimanana \& P. Vérin, sur «Andriamalala»p. 151, «Dox» p. 1013, «Estine Rinara Soa » p. 1137, « Ny Avana Ramanantoanina » p. 2611, «Rabearivelo » p. 3071, «Rado », «Rafanoharana» p. 3085, "Rainizanabololona p. 3088, "Rajaobelison ", "Rajaofera ", "Rajaonah » p. 3089, "Randriamiadanarivo ", "Randza Zanamihoatra » p. 3104, «Rapatsalahy» p. 3106, «Rasamuel » p. 3108, "Ratsifandrihamanana », «Ravelomoria » p. 3113, «Razafimahefa », «Razafiniaina » p. 3116 ; P. VÉRIN sur «Rajonah » p. 3090, «Ravelojaona » p. 3113, «Rodlish» p. 3220.]

Domenichini-Ramiaramanana Bakoly, 1968, Hainteny d'autrefois, poèmes traditionnels malgaches recueillis au début du règne de Ranavalona Ire, 1828-1861. Haintenin'ny fahiny... Tananarive, Librairie Mixte.

-, 1983, Du Ohabolana au Hainteny. Langue, littérature et politique à Madagascar, Paris, Karthala.

EsoAVElomandroso Faranirina V., 1976, «Langue, culture et colonisation à Madagascar : malgache et français dans l'enseignement officiel (1916-1940) ", Omaly sy Anio, 34, 1976, pp. 105-165.

GuEUNIER Noël J., 1996, «La Langue du catéchisme malgache de 1657. Les premiers essais d'une formulation malgache de la doctrine chrétienne ", in Le Christianisme dans le sud de Madagascar. Mélanges à l'occasion de la reprise de l'évangélisation dans le sud de Madagascar par la Congrégation de la Mission (Lazaristes), 18961996, Fianarantsoa, Ambozontany, pp. 23-77. 
-, 1997, "Le Catéchisme de Flacourt comme témoin des relations linguistiques dans le Sud-Est de Madagascar au milieu du XvII ${ }^{\mathrm{e}}$ siècle ", Études Océan Indien, 23-24, pp. 67-147. [Contient : «Lexique du Catéchisme de 1657», pp. 86-145]

-, 2004, "Expressions bibliques en malgache parlé contemporain ", in Rossi-Gensane N., (éd.), Mélanges en l'honneur de Nicole Gueunier, Tours, Université François Rabelais, pp. 273-298.

Gueunier Noël J., Rasoloniaina Brigitte, 2005-2006, «Les Cantiques malgaches de Dalmond (1841). Un des premiers essais de composition poétique et musicale dans l'Église catholique à Madagascar ", Études Océan Indien, 37, pp. 111-173.

Gueunier Noël J., Solo-Raharinjanahary, 2007, «Plantes de la Bible en malgache, et plantes "bibliques” en malgache. Quelques directions de recherche ", Communication au colloque Fankalazana ny faha-120 taonan'ny Fanitsiana Lehibe ny Baiboly Malagasy voalohany (120 e anniversaire de la Grande Révision de la Bible malgache), Fikambanana Mampiely Baiboly Malagasy, Antananarivo, 3-8 mai ; à paraître dans Études Océan Indien.

Huyghues-Belrose Vincent, 2001, Les premiers missionnaires protestants de Madagascar (1795-1827), Paris, Karthala.

KINGDON Abraham, ca. 1878, William Carey sy ny namany. Lektora efatra natao ny Abraham Kingdon teo anatrehan'ny Fikambanan'ny Tovolaby Kristiana eto Antananarivo, [William Carey et ses compagnons. Quatre conférences données devant l'Association des Jeunes gens chrétiens de Tananarive.] Antananarivo, Ny Friends' Foreign Mission Association.

LARSOn Pier M., 2000, History and Memory in the Age of Enslavement. Becoming Merina in Highland Madagascar, 1770-1822, Portsmouth-Oxford, Heinemann-James Currey.

MunTHE Ludvig, 1969, La Bible à Madagascar. Les deux premières traductions du Nouveau Testament malgache, Oslo, Egede Instituttet.

Munthe Ludvig, Ravaojanahary Charles, Ayache Simon, 1976, "Radama Ir et les Anglais : les négociations de 1817 d'après les sources malgaches (sorabe inédits) ", Omaly sy Anio, 3-4, pp. 9-104.

NoIRet François, 2008, Le Mythe d'Ibonia, le grand Prince (Madagascar), Paris, Karthala.

RaISON-Jourde Françoise, 1991, Bible et pouvoir à Madagascar au XIX siècle. Invention d'une identité chrétienne et construction de l'État (1780-1880), Paris, Karthala.

Rakotomalala Malanjaona, Blanchy Sophie, Raison-Jourde Françoise, (éds.), 2001, Usages sociaux du religieux sur les Hautes Terres malgaches. Les ancêtres au quotidien, Paris, L'Harmattan.

RAMAROSON Léonard, 1969, « Anaran'olona sy Anaran-tany ao amin'ny Testamenta Vaovao (Tantara kely) » [Noms de personnes et noms de lieux dans le Nouveau Testament (Un petit historique)], Tahiry sy Remby, 26, pp. 198-216.

Razafindrakoto Georges A., 2006, Old Testament Texts in Malagasy Contexts: An Analysis of the Use of the Old Testament in Three Religious Contexts in Madagascar, Thèse de Théologie, University of South Africa. Consultable en ligne : etd.unisa.ac.za/ ETD-db/theses/available/etd-08102006-113454/unrestricted/thesis.pdf

Smalley, William A., 1991, Translation as Mission. Bible Translation in the Modern Missionary Movement, Macon (Ga), Mercer University Press. 
SPINDLER Marc, 1992, "L'usage de la Bible dans le discours politique malgache depuis l'indépendance, 1960-1990 ", in Durand J.-D., Ladous R., (dirs.), Histoire religieuse, histoire globale, histoire ouverte. Mélanges offerts à Jacques Gadille, Paris, Beauchesne, pp. 199-220.

\section{Principales traductions malgaches de la Bible}

- La première traduction protestante, 1835 :

Ny Teny n'Andriamanitra, atao hoe, Baiboly Masina, misy ny Tesitamenta taloha sy taoriana, voadikia tamy ny teny Hebirio sy Girika; ka nafindra ny ny Misionary, avy amy ny "London Missionary Society, " ho teny Malagasy. [ La Parole de Dieu, qui est dite la Sainte Bible, avec le Testament ancien, et l'ultérieur, traduite des langues hébraïque et grecque ; et transposée par les missionnaires de la London Missionary Society en langue malgache. »] Antananarivo, Poresy ny ny London Missionary Society, 1835, pag. mult. (Réimpression fac-simile, 1985.)

Le Nouveau Testament, publié d'abord à part, a une page de titre particulière : $N y$ Teny n'Andriamanitra, atao hoe, Tesitamenta'ny Jesosy Kraisty Tompo'ntsika, sady Mpamonjy no Mpanavotra: No dikai'ny ny Misionary tamy ny teny Girika bo teny Malagasy, ka nampitovi'ny sy no dinihi'ny tamy ny teny sasany vao adika. [ La Parole de Dieu, qui est dite Testament de Jésus Christ notre Seigneur, ainsi que Sauveur et Libérateur : traduite par les missionnaires de la langue grecque, et accordée [ou : "rendue pareille"] et réfléchie avec d'autres langues avant d'être traduite »]

- La première révision protestante, dite de Griffiths :

Ny Baiboly, izany hoe, ny Soratra Masina rehetra amy ny Faneken-taloha sy ny Fanekem-baovao. ["La Bible, c'est-à-dire l'Ecriture Sainte entière, avec le Pacte ancien et le Pacte nouveau. »] Londona, W. M. Watts, 1865, s.p.

Le Nouveau Testament avait été imprimé, dès 1855, avec une page de titre différente : Ny Teniny ny Fanekem-baovao, milaza any Iesio Kiraitsy, Mpamonjy, Tompontsika. Voa dikiany ny Misionary tamy ny teny Girika, teo am-boalohany ary voa dinikia, ka voa hitsiny $D$. Griffiths, ambany ny anankiray mitandriny ny fandikiany ny Soratra Masina, teo aoriana. ["Les Paroles du Pacte nouveau, qui annoncent Jésus Christ, Sauveur et Seigneur. Traduites par les missionnaires de la langue grecque, à l'origine et étudiées, et corrigées ensuite par D. Griffiths, ensemble avec l'un des responsables de la traduction de l'Ecriture Sainte. »] Londona, W. M. Watts, 1855.

- La version révisée en 1887, puis en 1908-1909, devenue version reçue des Églises protestantes :

Ny Soratra Masina dia ny Testamenta taloha sy ny Testamenta vaovao. Nadika avy amin'ny teny Hebreo sy grika. [ L'Ecriture Sainte, c'est-à-dire l'Ancien Testament et le Nouveau Testament. Traduits des langues hébraïque et grecque. »] Tananarive, Fikambanana Mampiely Baiboly, 1965, 883-262 p. (La Sainte Bible en malgache, réimprimée de l'édition 1908-1909.)

- La traduction catholique :

Ny Baiboly na ny Soratra Masina. [ La Bible, ou L'Ecriture Sainte. »] Roma, Tipografia Poliglotta Vaticana, 1938, XVIII-1486 p. 
Au verso de la page de titre: Ny Baiboly Masina, nadikan'ny Mompera Jesoita amin'ny Vikarian'Antananarivo ho teny Malagasy [" La Sainte Bible, traduite par les Pères Jésuites du Vicariat d'Antananarivo en langue malgache. »]

Une révision est parue en 2006, éditée par la Société Biblique Malgache, Antananarivo, Fikambanana Mampiely Baiboly, v-1501-342 p.

- La traduction interconfessionnelle, ou œcuménique :

Ny Baiboly DIEM. Testamenta Taloha sy Testamenta Vaovao, miaraka amin'ny Boky Deoterôkanônika na Apôkrifa. Nadika avy amin'ny teny hebreo sy grika ho amin'ny teny malagasy of isialy ankehitriny. [" La Bible DIEM ("Traduction Commune de Madagascar”). Ancien Testament et Nouveau Testament, avec les livres deutérocanoniques ou apocryphes. Traduite des langues hébraïque et grecque en langue malgache officielle d'aujourd'hui. »] Antananarivo, Fikambanana Mampiely Baiboly Malagasy, 2005, XXIX-1251-II-251-420 p.

Le Nouveau Testament avait été imprimé dès 1991.

\section{Résumé}

La traduction de la Bible a exercé une influence profonde sur la constitution et la fixation d'une langue malgache moderne. Le processus de scripturalisation a commencé dans une période missionnaire, qui a coïncidé avec la formation d'un pouvoir d'État (1818-1895). L'histoire des traductions et révisions de la Bible en malgache montre la place fondatrice qu'a occupée l'entreprise de traduction protestante pour la langue nationale. Les choix faits alors n'ont plus été remis en cause; ils se retrouvent dans la traduction catholique (1938) et inspirent encore la traduction œecuménique (1991-2005). Toutes restent fidèles à un "langage biblique " désormais fixé. C'est sans doute pourquoi la traduction ocuménique n'a pas reçu un accueil très favorable. Paradoxalement, le recul de l'œecuménisme institutionnel se concilie avec l'association des deux traditions, protestante et catholique, fréquente dans les occasions coutumières. Là où le sociologue pouvait être tenté de chercher un syncrétisme, on voit plutôt une association et une négociation entre des traditions qui restent distinctes.

Mots-clés : Madagascar, langue nationale, écritures saintes, mission, traduction.

\section{Abstract}

Bible translation has had a profound effect on the constitution and fixation of modern Malagasy language. The writing process began to be successful in a missionary period contemporary with the formation of a State power (1818-1895). The history of the translations and revisions of the Malagasy Bible shows how fundamental was the protestant work of translation for the national language. Choices made then persist in the Catholic version (published 1938) and even in the ocumenical translation (1991-2005). They all retain a "biblical language" now fixed. This may explain why the recent inter-confessional translation has not been well received. Paradoxically, as institutional cecumenism recedes, the two traditions, Protestant and Catholic often join together in customary events. Where the sociologist might be tempted to seek a syncretism, there appears to be rather an association and a negotiation between distinct traditions.

Key words: Madagascar, national language, sacred scriptures, mission, translation. 


\section{Resumen}

La traducción de la Biblia ha ejercido una influencia profunda sobre la constitución y la fijación de una lengua malgache moderna. El proceso de puesta en escritura comenzó en un período misionero, que coincidió con la formación de un poder de Estado (1818-1895). La historia de las traducciones y revisiones de la Biblia en malgache muestra el lugar fundador que ha ocupado la empresa de traducción protestante en el idioma nacional. Las elecciones hechas entonces no fueron luego cuestionadas; podemos encontrarlas en la traducción católica (1938), e inspiran aún la traducción ecuménica (1991- 2005). Todas permanecen fieles a un "lenguaje bíblico" ya fijado. Paradójicamente, el retroceso del ecumenismo institucional se concilia con la asociación de las dos tradiciones, protestante y católica, frecuente en el las costumbres. Alli donde el sociólogo podría estar tentado de encontrar un sincretismo, vemos más bien una asociación y una negociación entre tradiciones que permanecen distintas. (trad. Véronica Giménez Béliveau)

Palabras clave: Madagascar, lengua nacional, santas escrituras, misión, traducción. 